\title{
Reduction in the energy loss of 0.5-MeV-per-atom carbon-cluster ions in thin carbon foils
}

\author{
S. Tomita, ${ }^{1,}{ }^{*}$ M. Murakami, ${ }^{1}$ N. Sakamoto, ${ }^{2, \dagger}$ S. Ishii, ${ }^{3}$ K. Sasa, ${ }^{3}$ T. Kaneko, ${ }^{4}$ and H. Kudo ${ }^{1}$ \\ ${ }^{1}$ Institute of Applied Physics, University of Tsukuba, Tsukuba, Ibaraki 305-8573, Japan \\ ${ }^{2}$ Department of Physics, Nara Women's University, Nara 630-8506, Japan \\ ${ }^{3}$ Tandem Accelerator Complex, University of Tsukuba, Tsukuba, Ibaraki 305-8577, Japan \\ ${ }^{4}$ Department of Applied Physics, Okayama University of Science, 1-1 Ridai-cho, Okayama 700-0005, Japan
}

(Received 20 May 2010; published 29 October 2010)

\begin{abstract}
We have precisely measured the energy loss of carbon cluster ions $\mathrm{C}_{n}{ }^{+}$with a kinetic energy of $0.5 \mathrm{MeV} /$ atom. The measured energies, lost in a thin carbon foil by cluster ions, are lower than that of an atomic ion with the equivalent speed $\left[\Delta E\left(\mathrm{C}_{n}{ }^{+}\right) / n<\Delta E\left(\mathrm{C}^{+}\right)\right]$, whereas most existing experimental results show the opposite trend with kinetic energies higher than $2.0 \mathrm{MeV}$ /atom. The experimental values are compared with the calculated ones, which take into account the effect of the Coulomb force, polarization force, and reduction in the cluster average charge on the response of a solid. The agreement between the measured and calculated energy losses indicates that the reduction in the energy loss is due to a structural correlation in electron excitation during the passage of the cluster.
\end{abstract}

DOI: 10.1103/PhysRevA.82.044901

When swift molecular ions are injected into a thin carbon foil, the energy loss of constituent individual atoms differs from the case of mono-atomic ion injection. This effect is known as a vicinage effect and was first reported by Brandt et al. [1] in 1974. Since then, several measurements of the energy loss of different diatomic molecular projectiles, such as $\mathrm{O}_{2}{ }^{-}$[2] $\mathrm{N}_{2}{ }^{+}$, and $\mathrm{O}_{2}{ }^{+}$[3] have been reported. The vicinage effect has been understood as a result of the coherent dynamic response of the target electrons because of the charged particles in tight clusters $[1,4]$. Most studies have shown that the energy lost by atomic ions dissociated from the molecular projectile was higher than that of atomic ions with equivalent speed. The opposite result was obtained only for limited cases of diatomic $\mathrm{N}_{2}{ }^{+}$and $\mathrm{O}_{2}{ }^{+}$ions running parallel to the interatomic axis [3].

In the 1990s, experimental investigations of the vicinage effect were applied to more complex large molecules such as $\mathrm{H}_{n}{ }^{+}$[5], $\mathrm{C}_{n}{ }^{+}$[6-8], and $\mathrm{B}_{n}{ }^{+}$[9]. Intensive experimental efforts were made, especially for carbon clusters. The energy of the projectiles ranges from 0.35 to $5.7 \mathrm{MeV}$ /atom. Most studies have shown that the vicinage effect is constructive, that is, the energy loss per atom for cluster ions is larger than that of an atomic ion $\left[\Delta E\left(\mathrm{C}_{n}{ }^{+}\right) / n>\Delta E\left(\mathrm{C}^{+}\right)\right]$. This tendency is stronger at higher injection energies. A larger cluster has a higher energy loss, but the effect of the enhanced energy loss is weaker [6]. This general behavior can be reproduced by calculations based on the interference with the response of the target electrons $[10,11]$. However, in the low-energy region, below $1 \mathrm{MeV} /$ atom, Kaneko [10] predicted a destructive effect, while Heredia-Avalos et al. [11] reported a constructive effect in the calculations. Only two experimental results for this energy region have been reported $[7,8]$. The experimental result reported by Brunelle et al. [8] showed a destructive effect, whereas Tomaschko et al. [7] showed a slightly constructive trend. In both cases, the uncertainty

\footnotetext{
*tomita@bk.tsukuba.ac.jp

$\dagger$ deceased
}

PACS number(s): $34.50 . \mathrm{Bw}, 36.40 .-\mathrm{c}, 78.70 .-\mathrm{g}$

is too large to allow for the discussion of such a small effect.

In previous experiments solid state detectors (SSD's) have been used to measure the kinetic energy of the projectile ions. Experimental difficulties in obtaining precise energy-loss data comes mainly from the response of the SSD to the molecular ions. Since the response depends on electronic stopping, the vicinage effect on the signal must be taken into account. Furthermore, a pulse height defect is also reported for the molecular beams [12]. Thus, kinetic-energy measurements of molecular ions with an SSD include a large uncertainty relative to the weak vicinage effect.

In the present Brief Report, we report precise measurements of the vicinage effect on carbon cluster ions with the kinetic energy of a $0.5 \mathrm{MeV} /$ atom. The experiments used the Rutherford backscattering (RBS) technique together with a sophisticated technique of energy-loss measurement developed at Nara Women's University $[13,14]$. The obtained experimental data were compared with the theoretical result.

Experiments were conducted at the Tandem Accelerator Complex at the University of Tsukuba. Negatively charged cluster ions were produced with a Cs sputter ion source and extracted with a potential difference of $20 \mathrm{kV}$. The extracted ions were mass selected by a $30^{\circ}$ magnet and injected into the $1 \mathrm{MV}$ tandem accelerator. In the accelerator, electrons were stripped and the positively charged cluster ions were accelerated to an energy of $0.5 \mathrm{MeV} /$ atom. The accelerated cluster ions were mass selected by deflecting them through $11^{\circ}$ with a magnet and finally injecting them into a scattering chamber in which the energy loss was measured.

A schematic drawing of the experimental setup is shown in Fig. 1. The accelerated cluster ions were impinged on a thin carbon foil after passing through an aperture of $1 \mathrm{~mm}$ in diameter. The carbon foil was mounted on a mechanical system together with an empty frame, which moved periodically so that the carbon cluster beam penetrated the carbon foil and the empty frame alternately. The frequency of the system was several $\mathrm{Hz}$, which is high enough to eliminate the 


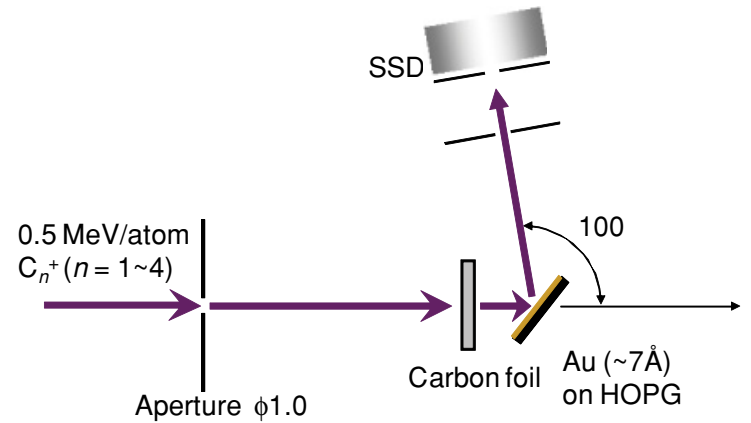

FIG. 1. (Color online) Schematic drawing of the experimental setup.

uncertainties that occurred due to the stability of the electronics [14]. To analyze the kinetic energy, the ions penetrating the foil were directed to a second target consisting of a thin $\mathrm{Au}$ layer evaporated on highly oriented pyrolytic graphite (HOPG). The energy of carbon atoms elastically scattered by $\mathrm{Au}$ atoms was measured at $100^{\circ}$ by an ion-implanted-silicon charged-particle detector (ORTEC ULTRA). The measured energy was proportional to the incident energy on the Au target, so the energy of the carbon atoms after penetration through the thin carbon foil can be measured. All the carbon clusters were dissociated during scattering therefore any vicinage effect in the pulse height defect can be avoided. The thickness of Au, measured by RBS, is $\sim 7 \AA$, which is thin enough so that the energy loss in the second target is negligible compared to that in the thin carbon foil.

The pulse height of the energy signal from the detector was analyzed by an analog-to-digital converter (ADC) and histogrammed separately according to the existence of the thin carbon foil. During the measurements, pulses from a pulse generator with two different pulse height settings were recorded simultaneously to ensure the stability of the electrical circuits. All signals were converted by the same ADC to eliminate the uncertainty due to the calibration of different ADC chips. The energy of the incident carbon clusters $\mathrm{C}_{n}{ }^{+}$ ( $n=1$ to 4 ) was $0.5 \mathrm{MeV} /$ atom. The amorphous carbon foil was purchased from Arizona Carbon Foil Co.; its thickness was determined to be 5.7 and $18.4 \mu \mathrm{g} / \mathrm{cm}^{2}$ from the energy loss of $0.5 \mathrm{MeV} \mathrm{C}^{+}$using the stopping power calculated by SRIM. The vacuum pressure was about $1 \times 10^{-6} \mathrm{~Pa}$ during the experiments. Energy calibrations were done by evaluating the peak positions of $\mathrm{C}^{+}$with energies of $0.24,0.50$, and $0.76 \mathrm{MeV}$.

Typical energy spectra obtained for $\mathrm{C}_{4}{ }^{+}$are shown in Fig. 2 . An energy shift due to the passage through the thin carbon foil is clearly visible. The spectra have nearly Gaussian shapes with widths of about $\pm 22 \mathrm{keV}$, which is mainly owing to the energy resolution of the SSD for low-energy carbon atoms. The standard deviation of the scattering angle of penetrated carbon atoms is estimated using TRIM code, which leads to the standard deviation of scattering energy of $\pm 1.1 \mathrm{keV}$ for a carbon foil of $5.7 \mu \mathrm{g} / \mathrm{cm}^{2}$. Together with the estimation of energy straggling of $5.9 \mathrm{keV}$, the estimation of the width of the energy spectra is $\pm 22.8 \mathrm{keV}$, which is almost the same as the spectra of the incident beam as seen in Fig. 2. The same

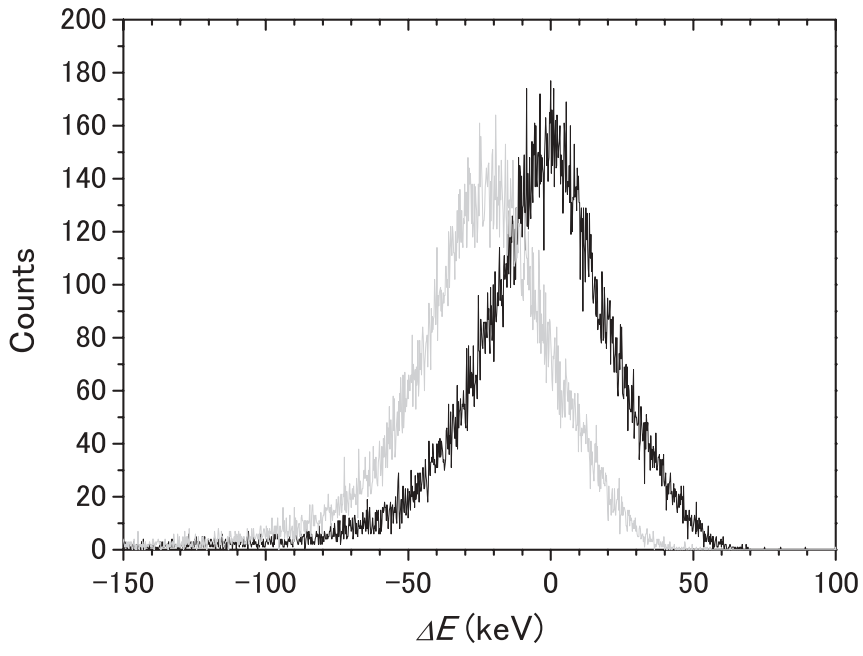

FIG. 2. Typical energy spectra obtained with foil (gray line) and without foil (black line) for $\mathrm{C}_{4}{ }^{+}$. Foil thickness was $5.7 \mu \mathrm{g} / \mathrm{cm}^{2}$.

estimation on $18.7 \mu \mathrm{g} / \mathrm{cm}^{2}$ gives the width of $\pm 23.4 \mathrm{keV}$. Therefore the peak shift $\delta$ between two spectra, $y_{1}(i)$ and $y_{2}(i)$, was determined by giving a minimum of

$$
\chi^{2}(\delta)=\sum_{i}\left\{\frac{y_{1}(i)-a y_{2}(i+\delta)}{\sigma(i)}\right\}^{2},
$$

with an appropriate $a$, where $\sigma(i)$ is evaluated from statistical errors. A rough estimate of the accuracy is given by $\delta E / \sqrt{n}$, where $\delta E$ is the energy width of the spectrum and $n$ is the number of measured particles. In the present case, we accumulate about 40000 particles, therefore the standard error in the peak position is of the order of $0.1 \mathrm{keV}$, which actually agrees with the standard deviation of our present data.

The difference of energy losses determined for $\mathrm{C}_{2}{ }^{+}$and $\mathrm{C}_{4}{ }^{+}$is shown in Table I. The difference of $\mathrm{C}_{4}+$ in $5.7 \mu \mathrm{g} / \mathrm{cm}^{2}$ is about $4 \%$ of the total energy loss $(22.7 \mathrm{keV})$ and decreases to $2 \%$ for $18.7 \mu \mathrm{g} / \mathrm{cm}^{2}$. The absolute value is about $1 \mathrm{keV}$ and almost the same for the two carbon foils; thus, the vicinage effect occurs within a thickness of $5.7 \mu \mathrm{g} / \mathrm{cm}^{2}$ from the surface. This is quite different from the vicinage effect observed for the yield of the secondary electrons [15-21]. The vicinage effect on the yield of the secondary electrons in the forward direction was reported to persist for much thicker targets such as $13 \mu \mathrm{g} / \mathrm{cm}^{2}$ for $\mathrm{H}_{n}{ }^{+}$[17] and $20 \mu \mathrm{g} / \mathrm{cm}^{2}$ for $\mathrm{C}_{n}{ }^{+}$[15]. Some mechanism other than the reduction in energy loss is evidently required to explain the strong reduction in the secondary electron yield. In this sense, the present result agrees with the possible vicinage effect on

TABLE I. Difference in energy loss $D=\Delta E\left(\mathrm{C}_{n}{ }^{+}\right) / n-$ $\Delta E\left(\mathrm{C}^{+}\right)$measured for two different foil thicknesses.

\begin{tabular}{ccc}
\hline \hline & $5.70 \mu \mathrm{g} / \mathrm{cm}^{2}$ & $18.4 \mu \mathrm{g} / \mathrm{cm}^{2}$ \\
\hline $\mathrm{C}_{2}{ }^{+}$ & $-0.90 \pm 0.11 \mathrm{keV}$ & $-0.71 \pm 0.33 \mathrm{keV}$ \\
$\mathrm{C}_{4}{ }^{+}$ & $-1.07 \pm 0.39 \mathrm{keV}$ & $-1.44 \pm 0.45 \mathrm{keV}$ \\
\hline \hline
\end{tabular}




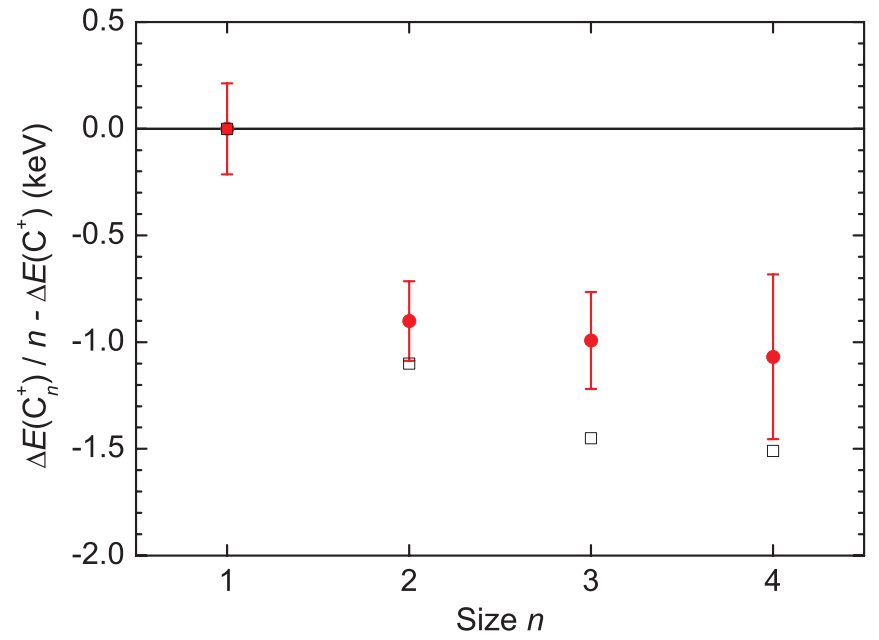

FIG. 3. (Color online) Energies lost by carbon cluster ions $\mathrm{C}_{n}{ }^{+}$ $(0.5 \mathrm{MeV} /$ atom $)$ penetrating a carbon foil of $5.7 \mu \mathrm{g} / \mathrm{cm}^{2}$ thickness, relative to that lost by atomic ions with equivalent speed. Calculated results (open squares) are plotted together with experimental values (filled circles).

the transport of scattered electrons recently suggested by Arai et al. [20].

The average energy shift obtained for the $\mathrm{C}_{n}{ }^{+}$incidence relative to that of the $\mathrm{C}^{+}$incidence is shown in Fig. 3, together with the results of the theoretical calculations. First, the average energy loss of the constituent atoms of the cluster ions is found to be about $1 \mathrm{keV}$ lower than in the case of atomic ion injection. This reduction becomes larger with increasing cluster size and seems to converge. The magnitude of the reduction is quite small, about $4 \%$ of the total energy loss. This agrees well with the speculation that the convoy electron yield by $\mathrm{C}_{n}{ }^{+}$is proportional to the electronic stopping [15].

As the figure shows, the experimental results are reproduced quite well by the present calculation in which the trajectory and average charge of each constituent ion of the cluster were determined [22], taking into account the repulsive Coulomb force and the polarization force in the dielectric media. The carbon clusters are assumed to be ensembles of isolated atoms in a linear chain structure with an equal spacing of $0.127 \mathrm{~nm}$. Also, they are assumed to be randomly oriented when injected into a solid, so they do not have any special alignment. The spatial distribution of the bound electrons is described by a Thomas-Fermi-Molière function with a size parameter that depends on the degree of ionization [10]. The average charge states of the ions in a cluster were determined by the self-consistent theory, where the charge state of a given ion was determined by its speed, the distances from surrounding ions, and the charge states of the surrounding ions. The ion speed was affected by the forces acting on it. We take into account the dynamic polarization force originating from the surrounding ions within a dielectric function treatment. A molecular dynamics calculation yields the trajectory, kinetic energy, and average charge of each ion step by step for a small time interval. Therefore, the energy loss and the charge state of the trailing ion is affected by those of the leading ion via the dynamic polarization force. The instantaneous electronic stopping cross section for the whole cluster is described in the dielectric function form as follows [10]:

$$
S=\frac{2}{\pi V^{2}} \int_{0}^{\infty} \frac{d k}{k^{2}} \int_{0}^{k V} d \omega \omega \operatorname{Im}\left[\frac{-1}{\varepsilon(k, \omega)}\right]\left\langle\left|\rho_{\text {ext }}(\vec{k})\right|^{2}\right\rangle,
$$

where $\epsilon(k, \omega)$ is the dielectric function of the medium and $V$ is the speed of the cluster moving in the material. The factor $\left\langle\left|\rho_{\text {ext }}(\vec{k})\right|^{2}\right\rangle$ is the orientation average of the square charge density of the cluster in Fourier space, obtained from $\rho_{\text {ext }}(\vec{r})=e \sum_{i}\left[Z \delta\left(\vec{r}-\overrightarrow{R_{i}}\right)-\rho_{i}\left(\vec{r}-\vec{R}_{i}\right)\right]$. We have the following expression,

$$
\begin{aligned}
\left\langle\left|\rho_{\mathrm{ext}}(\vec{k})\right|^{2}\right\rangle= & e^{2} \sum_{i}\left\{Z-\rho_{i}(k)\right\}^{2}+e^{2} \sum_{i} \sum_{j \neq i}\left\{Z-\rho_{i}(k)\right\} \\
& \times\left\{Z-\rho_{j}(k)\right\} \frac{\sin \left(k R_{i j}\right)}{k R_{i j}},
\end{aligned}
$$

where $R_{i j}$ is the relative distance between the $i$ th and $j$ th ions. Here $\rho_{i}(k)$ is the Fourier transform of the spherically averaged bound-electron distribution $\rho_{i}(\vec{r})$ of the $i$ th ion. The vicinage effect originates from the effective external charge in Fourier space, shown in Eq. (3). When the mutual separation becomes infinite, the effect of the external charge clearly reduces to a sum of the contributions of individual ions. This means that the vicinage effect vanishes. Whether the cluster effect becomes negative or positive depends on the second term of Eq. (3) (i.e., the charge correlation function for a momentum transfer $\hbar k)$.

The negative cluster effect observed in the present energyloss measurement demonstrates a mechanism based on the response of an electric gas in a solid. On the other hand, it

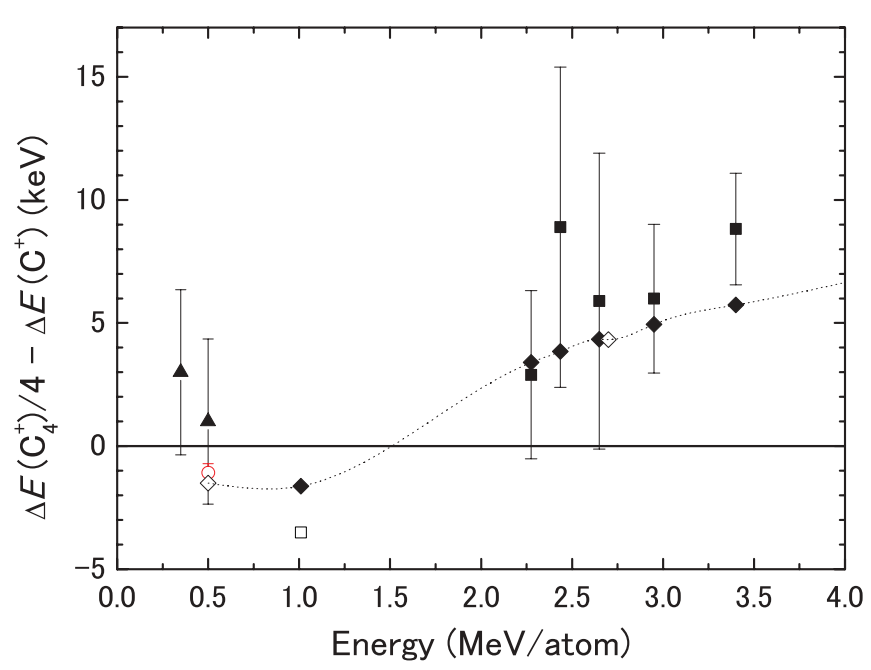

FIG. 4. (Color online) Difference in energies lost by carbon cluster ions $\mathrm{C}_{4}{ }^{+}$compared to that of $\mathrm{C}^{+}$with the same velocity as a function of injection energy. The present experimental result (open circle) and theoretical calculation (open diamonds) are shown with other experimental values of Baudin et al. [6] (filled squares), Brunelle et al. [8] (open square), and Tomaschko et al. [7] (filled triangles). Theoretical results from Ref. [10] are also shown for comparison (filled diamonds). Dotted line is shown as a guide to the eye. 
is interesting that the calculation reported by Heredia-Avalos et al. [11] does not show such a suppression effect. At the lowest energy \{i.e., $1.01 \mathrm{MeV} /$ atom for a $\mathrm{C}_{n}{ }^{+}(n=2-8)$ [11] $\}$ a positive value for $D=\Delta E\left(\mathrm{C}_{n}{ }^{+}\right) / n-\Delta E\left(\mathrm{C}^{+}\right)$was obtained. This is opposite to the experimental data of Brunelle et al. [8]. However, in the present treatment, negative values of $D$ were obtained again in a low-energy region (0.5 MeV/atom). The major difference in our calculation is that we consider the reduction in the charge state of individual constituent ions inside the foil and the spatial electron distribution in the Thomas-Fermi-Molière approximation, not in the Brandt-Kitagawa form [11]. In our calculation, the average charge of $\mathrm{C}$ is 1.48 and the average charge reduction amounts range from $5 \%\left(\mathrm{C}_{2}\right)$ to $9 \%\left(\mathrm{C}_{4}\right)$. We confirm that this negative cluster effect is smeared out from one-third to one-half if we neglect the reduction in the average charge.

The energy dependence of the vicinage effect is shown in Fig. 4, together with all the experimental results reported so far [6-8], and the previous theoretical calculation [10]. Our error bar is clearly extremely small compared to that of the other data. The error is mainly because of the degradation of the detector during the experiments. The observed deconstructive effect agrees with all the previously reported experimental data, considering their errors. The effect seems to be reversed around the velocity of $1 \mathrm{MeV}$ /atom for carbon cluster ions.

We have precisely measured the energy loss of carbon cluster ions $\mathrm{C}_{n}{ }^{+}(n=1-4)$ with a kinetic energy of $0.5 \mathrm{MeV} /$ atom in thin carbon foils. The measured energy lost by cluster ions is significantly lower than that of an atomic ion with the equivalent speed. The experimental values are adequately predicted by the present calculation in which the cluster average charge plays a significant role when the polarization, the Coulomb repulsion, the spatial structure, and the size of the electron clouds are included.

The authors thank the technical staff in the accelerator complex for their technical assistance. T.K. is grateful for a Grant-in-Aid for Scientific Research (C) from JSPS.
[1] W. Brandt, A. Ratkowski, and R. H. Ritchie, Phys. Rev. Lett. 33, 1325 (1974)

[2] J. W. Tape, W. M. Gibson, J. Remillieux, R. Laubert, and H. E. Wegner, Nucl. Instrum. Methods 132, 75 (1976).

[3] M. F. Steuer, D. S. Gemmell, E. P. Kanter, E. A. Johnson, and B. J. Zabransky, Nucl. Instrum. Methods Phys. Res. 194, 277 (1982).

[4] N. R. Arista, Phys. Rev. B 18, 1 (1978).

[5] E. Ray, R. Kirsch, H. H. Mikkelsen, J. C. Poizat, and J. Remillieux, Nucl. Instrum. Methods Phys. Res., Sect. B 69, 133 (1992).

[6] K. Baudin et al., Nucl. Instrum. Methods Phys. Res., Sect. B 94, 341 (1994).

[7] C. Tomaschko, D. Brandl, R. Kügler, M. Schurr, and H. Voit, Nucl. Instrum. Methods Phys. Res., Sect. B 103, 407 (1995).

[8] A. Brunelle, S. Della-Negra, J. Depauw, D. Jacquet, Y. Le Beyec, M. Pautrat, and C. Schoppmann, Nucl. Instrum. Methods Phys. Res., Sect. B 125, 207 (1997).

[9] K. Narumi, K. Nakajima, K. Kimura, M.-h. Mannami, Y. Saitoh, S. Yamamoto, Y. Aoki, and H. Naramoto, Nucl. Instrum. Methods Phys. Res., Sect. B 135, 77 (1998).

[10] T. Kaneko, Phys. Rev. A 66, 052901 (2002).

[11] S. Heredia-Avalos, R. Garcia-Molina, and I. Abril, Phys. Rev. A 76, 012901 (2007).

[12] M. Seidl, H. Voit, S. Bouneau, A. Brunelle, S. Della-Negra, J. Depauw, D. Jacquet, Y. Le Beyec, and M. Pautrat, Nucl. Instrum. Methods Phys. Res., Sect. B 183, 502 (2001).
[13] R. Ishiwari, N. Shiomi-Tsuda, and N. Sakamoto, Nucl. Instrum. Methods Phys. Res., Sect. B 31, 503 (1988).

[14] R. Ishiwari, N. Shiomi-Tsuda, N. Sakamoto, and H. Ogawa, Nucl. Instrum. Methods Phys. Res., Sect. B 47, 111 (1990).

[15] S. Tomita, S. Yoda, R. Uchiyama, S. Ishii, K. Sasa, T. Kaneko, and H. Kudo, Phys. Rev. A 73, 060901 (2006).

[16] N. V. de Castro Faria, B. Farizon Mazuy, M. Farizon, M. J. Gaillard, G. Jalbert, S. Ouaskit, A. Clouvas, and A. Katsanos, Phys. Rev. A 46, 3594(R) (1992).

[17] A. Billebaud, D. Dauvergne, M. Fallavier, R. Kirsch, J. C. Poizat, J. Remillieux, H. Rothard, and J. P. Thomas, Nucl. Instrum. Methods Phys. Res., Sect. B 112, 79 (1996).

[18] M. Fallavier, R. Kirsch, J. C. Poizat, J. Remillieux, and J. P. Thomas, Nucl. Instrum. Methods Phys. Res., Sect. B 164, 920 (2000).

[19] H. Kudo, W. Iwazaki, R. Uchiyama, S. Tomita, K. Shima, K. Sasa, S. Ishii, K. Narumi, H. Naramoto, Y. Saitoh, S. Yamamoto, and T. Kaneko, Jpn. J. Appl. Phys. Part 2-Lett. Exp. Lett. 45, L565 (2006).

[20] H. Arai, H. Kudo, S. Tomita, and S. Ishii, J. Phys. Soc. Jpn. 78, 104301 (2009)

[21] Y. Takahashi, K. Narumi, A. Chiba, Y. Saitoh, K. Yamada, N. Ishikawa, H. Sugai, and Y. Maeda, Europhys. Lett. 88, 63001 (2009).

[22] A. Chiba, Y. Saitoh, K. Narumi, M. Adachi, and T. Kaneko, Phys. Rev. A 76, 063201 (2007). 